\title{
Competencias de sostenibilidad ambiental para los alumnos del CUCEA
}

\author{
María Elena Peyro Beltrán ${ }^{1}$
}

\section{Resumen}

El objetivo de este trabajo consiste en encauzar el desarrollo de competencias en sostenibilidad ambiental en los alumnos del CUCEA con la finalidad de que adquieran conocimientos, habilidades y aptitudes para generar propuestas de intervención en contextos diferenciados dentro del marco de las crisis ambientales contemporáneas. Para lograr tal objetivo, se propone hacer uso de las cinco competencias aplicadas por Evans en un contexto universitario: competencia de sistemas; crítica y analítica; interpersonal y de comunicación; creativa y estratégica; y transdisciplinaria.

En consecuencia, se busca presentar una propuesta de práctica docente con ejemplificación de actividades pedagógicas con un giro transdisciplinario para la formación de tales competencias en materia de desarrollo sostenible.

Palabras clave: formación de competencias y pedagogías en sustentabilidad, educación para el desarrollo sostenible.

\section{Introducción}

Ante las crecientes crisis ambientales en el territorio mexicano, nos enfrentamos a diversos retos en materia de desarrollo sostenible, tales como: conservar la biodiversidad de fauna y flora; asegurar la disponibilidad y calidad del recurso hídrico; reducir sus emisiones de gases de efecto invernadero; avanzar en la adaptación a los efectos del cambio climático; y mejorar la calidad del aire en gran cantidad de zonas urbanas del país; entre otras (Semarnat, 2018; López-García y Manzano, 2016). de Desarrollo y Medio Ambiente Correo electrónico: maria.p@cucea.udg.mx 
En este contexto, los grandes desafíos alrededor de los problemas ecológicos y de desigualdad social demandan la toma de conciencia de diversos actores, entre ellos las instituciones de educación superior. Dado que, como espacios de formación de futuros profesionistas, su rol pedagógico resulta imprescindible para apuntalar competencias en materia de sostenibilidad de una manera estratégica. Por su parte, Nieto y Medellín (2005) señalan que:

El conocimiento que se construya en las instituciones de educación superior mexicanas, y la formación de profesionistas críticos y creativos, son indispensables para contribuir a una mejor comprensión de nuestras realidades, así como a generar propuestas concretas de cambio hacia la sustentabilidad y construcción de visiones alternativas de futuro (p. 40).

Por lo tanto, en este trabajo se muestra una propuesta de práctica docente universitaria con ejemplificación de competencias sostenibles y actividades pedagógicas con el fin de formar alumnos aptos para enfrentar diversas problemáticas ambientales en contextos situados con una perspectiva sistémica y transdisciplinaria.

Para lograr tal cometido, hago uso de la rigurosa investigación realizada por Evans (2019) sobre las competencias en desarrollo sostenible aplicadas en un contexto pedagógico de educación superior. A continuación presento la fundamentación detrás de tales competencias para consecuentemente exponer una propuesta práctica docente con orientación en desarrollo sostenible en el CUCEA.

\section{Competencias en desarrollo sostenible}

Sobre el concepto de competencia en materia educativa, Bronckart y Dolz (2007) refieren que su significado puede ser entendido como "toda capacidad adquirida por el saber o la experiencia” (p. 151). Así, la formación académica brinda a los alumnos conocimientos en competencias que son validados por el Estado, cuya certificación otorgada a los sujetos posibilita la adquisición de cierto cargo laboral. La variabilidad dentro del contexto profesional requiere una progresiva adaptación a nuevas metas, instrumentos e informaciones. Asimismo, de acuerdo con dichos autores, los conocimientos "estáticos" ya no bastan para formar a los próximos profesionales; en lugar de eso se puede concebir que se les forme desde capacidades generales y flexibles que les posibiliten adaptarse a los desafíos y tareas que puedan encontrar.

Por otro lado, Murga-Menoyo (2015) expone que debido a las demandas sociales urgentes derivadas de las problemáticas socioambientales, es de vital importancia que las competencias desarrolladas en los programas de educación formal incluyan estrategias pedagógicas transversales y diversificadas que permitan afrontar la crisis actual desde sus manifestaciones contextuales. De manera que la unESCo (2014) manifiesta que la formación de tales competencias debe incluir la orientación del desarrollo sostenible: 
La eds [educación en desarrollo sostenible] se refiere a los aspectos medioambientales, sociales y económicos del desarrollo sostenible de una manera integrada, equilibrada e integral. Se relaciona asimismo con una agenda global para el desarrollo sostenible como la que figura en el documento final de la Conferencia Río+20 que comprende, entre otras cosas, cuestiones interrelacionadas como la reducción de la pobreza, el cambio climático, la reducción de los riesgos de desastre, la biodiversidad y el consumo y la producción sostenibles. Responde a especificidades locales y respeta la diversidad cultural (p. 33).

Sobre la implementación de tales competencias en un contexto de educación superior, en el siguiente apartado se expone el modelo pedagógico en desarrollo sostenible de Tina Lynn Evans, cuyos avances se configuran como una ruta para diversos contextos institucionales.

\section{Modelo pedagógico de sostenibilidad de Evans}

A partir de una revisión extensa y rigurosa en producción académica, combinando el enfoque de teoría fundamentada para analizar temas/conceptos desde la interpretación hermenéutica, Evans (2019) propone un marco de referencia basado en competencias para los estudios de sostenibilidad, junto con pedagogías potenciales para enseñar tales habilidades efectivamente en las universidades.

En seguida se muestran los hallazgos resultantes de un mapeo transversal de las competencias centrales en la enseñanza de sostenibilidad, así como los enfoques pedagógicos aplicados en la Licenciatura de Artes en Estudios de Sostenibilidad de Colorado Mountain College.

\subsection{Niveles de competencias}

Evans (2019) propone la articulación de cinco competencias en constante convergencia dinámica para la promoción de conocimiento, habilidades y actitudes/orientaciones en sostenibilidad:

Competencia de sistemas:

"Capacidad para analizar colectivamente sistemas complejos en múltiples dominios (cultural, ambiental, económico, político, etc.) y en diferentes escalas (local a global) mediante la consideración de efectos en cascada, inercia, ciclos de retroalimentación, emergencia y otras características sistémicas para desarrollar conocimientos relacionados con problemas, desafíos y oportunidades de sostenibilidad (pasado, presente y futuro)" (p. 7).

Competencia crítica y normativa:

"Capacidad para crear, descubrir, experimentar, negociar, comunicar y activar colectivamente principios y objetivos orientados a la sostenibilidad” (p. 8).

Competencia interpersonal y de comunicación:

"Capacidad para habilitar, facilitar y motivar el aprendizaje, el pensamiento y la acción de sostenibilidad colaborativa y participativa” (p. 8). 


\section{Competencia creativa y estratégica:}

"Capacidad para visualizar, desarrollar, implementar y evaluar colectivamente intervenciones transformadoras para la sostenibilidad" (p. 9).

Competencia transdisciplinaria:

"Capacidad para basarse, de manera crítica e integradora, en múltiples marcos disciplinarios para informar el pensamiento y las acciones orientadas a la sostenibilidad. Implica alfabetización epistemológica: comprensión de múltiples formas de conocimiento, incluidas sus respectivas metodologías, aplicaciones, beneficios y limitaciones. Además, conlleva competencias de sostenibilidad de forma colaborativa e inclusiva para fomentar el cambio social" (p. 9).

\subsection{Pedagogías para el desarrollo de competencias}

Referente a los enfoques pedagógicos para enseñar las competencias previamente enlistadas, Evans (2019) propone los siguientes:

1. Aprendizaje basado en proyectos/problemas (en una organización/comunidad).

2. Aprendizaje integrativo (interdisciplinario y transdisciplinario).

3. Aprendizaje basado en proyectos/problemas (en clase).

4. Aprendizaje activo (en clase).

5. Aprendizaje de servicio.

6. Aprendizaje basado en la investigación.

7. Análisis/interpretación crítica de textos/información.

8. Aprendizaje reflexivo.

9. Aprendizaje colaborativo.

En consecuencia, un programa profesionalizante en las competencias de sostenibilidad, previamente desarrolladas, con tales pedagogías, se configuraría de la siguiente manera:

[...] un curso de aprendizaje activo basado en proyectos con un enfoque en la creación de conocimientos/aprendizajes integrativos; la colaboración intensiva en equipos de proyectos; y el compromiso de las partes interesadas. El curso también representaría una forma de aprendizaje de servicio, ya que atendería las necesidades de una organización, y requeriría una extensa investigación, así como análisis e interpretación de la información y las comunicaciones. El curso también requeriría una reflexión sobre las contribuciones propias y de los demás a los procesos colaborativos de gestión de proyectos (Evans, 2019: 18).

\section{Competencias de sostenibilidad en los alumnos de las diversas carreras del cucea}

Las competencias en sostenibilidad que propone el modelo de Evans (2019), es una ruta para dar inicio a la formación de competencias en sostenibilidad en los alumnos del CUCEA. 
Estas competencias implicarán una educación transversal de enseñanza en desarrollo sustentable en todas las licenciaturas del CUCEA de las carreras como Economía, Gestión y Economía Ambiental, Turismo, Administración, Negocios Internacionales, Políticas Públicas, etc., a través de la identificación, análisis y resolución de problemas ambientales por medio de la transdisciplinariedad en ámbitos nacionales y locales.

La siguiente propuesta de competencias de sostenibilidad ambiental para los alumnos del CUCEA sería el inicio para elaborar una estrategia conjunta en colaboración con los profesores del Centro interesados en formar alumnos con habilidades, competencias y actitudes en sostenibilidad ambiental a través de la impartición de la materia de Desarrollo Sustentable.

En seguida se muestra la ejemplificación de actividades pedagógicas orientadas al desarrollo de las competencias del modelo de Evans:

Competencia de sistemas:

- Habilidad: analizar sistemas complejos considerando diversos dominios (social, económico, político y ambiental).

- Actividad pedagógica.

- Análisis sistémico de un problema ambiental en lo social, económico, político y ambiental (contaminación y escasez de agua, contaminación atmosférica, incendios, deforestación, residuos urbanos, residuos peligrosos, basura, etcétera.

- Investigación/proyecto problemática ambiental global y su efecto local (cambio climático, escasez de agua, pandemias, migraciones, etcétera).

- Elaboración de escenarios ambientales (agua, aire, suelos, deforestación, desechos peligrosos, biodiversidad (ecosistemas, especies), etc., país, estatal, local, o comunitaria.

Competencia crítica y normativa:

- Habilidad: formular preguntas sobre las causas de los problemas para resolverlos.

- Actividad pedagógica.

- Debates problemas ambientales.

- Trabajo en equipos transdisciplinarios para análisis y gestión de problemáticas ambientales.

Competencia Interpersonal y de comunicación:

- Habilidad: capacidad para colaborar y negociar.

- Actividad pedagógica.

- Comunicación y negociación con los diferentes actores (Estado, empresas, sociedad, academia) de proyectos ambientales o resolución de problemas socioambientales.

Competencia creativa y estratégica:

- Habilidad: identificar factores que permiten o impiden un cambio orientado a la sostenibilidad y formular estrategias en torno a éstas.

- Actividad pedagógica.

- Elaboración de proyectos colectivos ambientales y de intervención. 
- Creación de estrategias creativas y de innovación para la resolución de problemáticas ambientales.

Competencia transdisciplinaria:

- Habilidad: integrar grupos multidisciplinarios para abordar la complejidad de una problemática socioambiental.

- Actividad pedagógica.

- Conformación de equipos multidisciplinarios para resolución de problemas ambientales locales y nacionales.

- Análisis de estudios de casos.

\section{Reflexión final}

Con este trabajo se buscó encauzar una iniciativa de desarrollo de competencias sostenibles en los alumnos del CUCEA, con el fin de que adquieran conocimientos, habilidades y actitudes para generar propuestas de análisis, investigación, intervención y solución a problemas ambientales locales, estatales y nacionales con una perspectiva sistémica y transdisciplinaria. Ello implicaría la enseñanza de desarrollo sustentable y sustentabilidad en las diversas carreras de nuestro Centro Universitario.

Para lograr tal objetivo se propuso hacer uso de las cinco competencias aplicadas por Evans en un contexto universitario: competencia de sistemas; crítica y analítica; interpersonal y de comunicación; creativa y estratégica; y transdisciplinaria a través de un ensayo de práctica docente con ejemplificación de actividades pedagógicas.

El mayor reto sería la creación de una cultura ambiental en CUCEA, ya que se necesitaría del consenso y participación de los profesores, coordinadores y del apoyo institucional del Centro y la misma Universidad.

Así, la formación de competencias propuestas requiere la capacitación de los profesores en su práctica docente en sostenibilidad.

\section{Referencias bibliográficas}

Bronckart, J. P., y Dolz-Mestre, J. (2007). La noción de competencia: Su pertinencia para el estudio del aprendizaje de las acciones verbales. Desarrollo del lenguaje y didáctica de las lenguas (pp. 147-165). Buenos Aires: Miño y Dávila.

Evans, L. T. (2019). Competencies and Pedagogies for Sustainability Education: A Roadmap for Sustainability Studies Program Development in Colleges and Universities. Sustainability, 11(19): 5526. doi: 10.3390/su11195526.

López-García, T. G., y Manzano, M. G. (2016). Vulnerabilidad climática y situación socioambiental: Percepciones en una región semiárida del noreste de México. Madera y Bosques, 22(2): 105-117.

Murga-Menoyo, M. A. (2015). Competencias para el desarrollo sostenible: Las capacidades, actitudes y valores meta de la educación en el marco de la Agenda Glo- 
bal post-2015. Foro de Educación, 13(19): 55-83. doi: http://dx.doi.org/10.14516/ fde.2015.013.019.004.

Nieto, L. M., y Medellín, P. (2007). Medio ambiente y educación superior: Implicaciones en las políticas públicas. Revista de la Educación Superior, 36(142): 21-42.

Semarnat. (2018). Informe del medio ambiente en México. Recuperado de https:// apps1.semarnat.gob.mx:8443/dgeia/informe18/index.html

Unesco. (2014). Hoja de ruta la ejecución del Programa de Acción Mundial de Educación para el Desarrollo Sostenible. Recuperado de https://unesdoc.unesco.org/ ark:/48223/pf0000230514 